PROCEEDINGS OF THE

AMERICAN MATHEMATICAL SOCIETY

Volume 134, Number 10, October 2006, Pages 2931-2941

S 0002-9939(06)08421-8

Article electronically published on April 11, 2006

\title{
ON GENERALIZED HYPERINTERPOLATION ON THE SPHERE
}

\author{
FENG DAI
}

(Communicated by Andreas Seeger)

\begin{abstract}
It is shown that second-order results can be attained by the generalized hyperinterpolation operators on the sphere, which gives an affirmative answer to a question raised by Reimer in Constr. Approx. 18(2002), no. 2, $183-203$.
\end{abstract}

\section{INTRODUCTION}

Let $\mathbb{S}^{d-1}=\left\{x \in \mathbb{R}^{d}: \quad|x|=1\right\}(d \geq 3)$ be the unit sphere of $\mathbb{R}^{d}$ endowed with the usual rotation invariant measure $d \sigma(x)$. We denote by $\mathcal{H}_{k}^{d}$ the space of all spherical harmonics of degree $k$ on $\mathbb{S}^{d-1}$ and $\Pi_{N}^{d}$ the space of all spherical polynomials of degree at most $N$. The spaces $\mathcal{H}_{k}^{d}$ are mutually orthogonal with respect to the inner product

$$
\langle f, g\rangle=\int_{\mathbb{S}^{d-1}} f(x) g(x) d \sigma(x),
$$

and the space $\Pi_{N}^{d}$ can be expressed as a direct sum

$$
\Pi_{N}^{d}=\mathcal{H}_{0}^{d} \oplus \mathcal{H}_{1}^{d} \oplus \cdots \oplus \mathcal{H}_{N}^{d} .
$$

Also, it is known that for $f \in \mathcal{H}_{k}^{d}$,

$$
f(x)=\int_{\mathbb{S}^{d-1}} f(y) G_{k}(x \cdot y) d \sigma(y), \quad x \in \mathbb{S}^{d-1},
$$

where here and throughout,

$$
G_{k}(t)=\frac{2 k+d-2}{(d-2)\left|\mathbb{S}^{d-1}\right|} P_{k}^{\frac{d-2}{2}}(t), \quad t \in[-1,1],
$$

$\left|\mathbb{S}^{d-1}\right|$ is the surface area of $\mathbb{S}^{d-1}$, and $P_{k}^{\frac{d-2}{2}}(t)$ is the usual ultraspherical polynomial of degree $k$ and index $\frac{d-2}{2}$, as defined in [Sz, p. 80]. Thus, by (1.1), we have, for $f \in \Pi_{N}^{d}$,

$$
f(x)=\int_{\mathbb{S}^{d-1}} f(y) E_{N}(x \cdot y) d \sigma(y), \quad x \in \mathbb{S}^{d-1},
$$

Received by the editors April 23, 2005.

2000 Mathematics Subject Classification. Primary 41A15, 41A17; Secondary 41A05, 46E22.

Key words and phrases. Spherical polynomials, generalized hyperinterpolation, second-order moduli of smoothness, unit sphere.

The author was supported in part by the NSERC Canada under grant G121211001.

(C)2006 American Mathematical Society Reverts to public domain 28 years from publication 
where

$$
E_{N}(x \cdot y)=\sum_{k=0}^{N} G_{k}(x \cdot y)
$$

We denote by $d(x, y)$ the geodesic distance $\arccos x \cdot y$ between $x$ and $y$ on $\mathbb{S}^{d-1}$, $B(x, r)$ the spherical cap $\left\{y \in \mathbb{S}^{d-1}: d(x, y) \leq r\right\}$ centered at $x \in \mathbb{S}^{d-1}$ of radius $r>0$, and $B(x, \alpha, \alpha+\beta)$ the spherical collar $\left\{y \in \mathbb{S}^{d-1}: \quad \alpha \leq d(x, y) \leq \alpha+\beta\right\}$ centered at $x \in \mathbb{S}^{d-1}$ of spherical height $\beta>0$. We shall use the notation $|E|$ to denote the measure $\sigma(E)$ of a subset $E \subset \mathbb{S}^{d-1}$, and write $A \sim B$ for the statement $C^{-1} A \leq B \leq C A$, where $C>0$ is called the constant of equivalence.

Throughout this paper, we assume that $\left\{Q_{N}\right\}_{N=0}^{\infty}$ is a sequence of positive cubature formulas on $\mathbb{S}^{d-1}$ :

$$
\int_{\mathbb{S}^{d-1}} f(x) d \sigma(x)=Q_{N} f:=\sum_{\omega \in \Lambda_{N}} \lambda_{N, \omega} f(\omega), \quad \text { for all } f \in \Pi_{N+1}^{d},
$$

where $\Lambda_{N}$ is a finite subset of $\mathbb{S}^{d-1}, \lambda_{N, \omega}>0$ for all $\omega \in \Lambda_{N}$. A cubature formula like $Q_{N}$ is said to be of degree $N+1$. It was shown by Reimer Re1 that a positive cubature formula $Q_{N}$ of degree $N+1$ on the sphere must satisfy the following regularity condition, introduced by Sloan and Womersley [SW]:

$$
\max _{x \in \mathbb{S}^{d-1}} \sum_{\omega \in \Lambda_{N} \cap B\left(x, N^{-1}\right)} \lambda_{N, \omega} \leq C N^{-(d-1)},
$$

where $C>0$ depends only on $d$.

The hyperinterpolation operators $L_{N}$ on $\mathbb{S}^{d-1}$, introduced by Sloan [Sl] (see also $[\mathrm{SW}]$ ), are defined as

$$
L_{N} f(x)=\sum_{\omega \in \Lambda_{2 N-1}} \lambda_{2 N-1, \omega} f(\omega) E_{N}(x \cdot \omega), \quad x \in \mathbb{S}^{d-1}, \quad f \in C\left(\mathbb{S}^{d-1}\right), N=1,2, \ldots,
$$

where the $E_{N}$ 's are defined by (1.3). The following remarkable result was proved by [SW] $(d=3$, under the regularity assumption (1.4) $)$, LS] ( $d$ arbitrary, under the regularity assumption (1.4), and by [Re1] ( $d$ arbitrary, no additional regularity assumption):

$$
\left\|L_{N}\right\|_{\infty} \sim N^{\frac{d-2}{2}},
$$

where the constant of equivalence depends only on $d$, and

$$
\left\|L_{N}\right\|_{\infty}:=\sup \left\{\left\|L_{N} f\right\|_{\infty}: \quad f \in C\left(\mathbb{S}^{d-1}\right)\right\} .
$$

We refer to [SW], LS], Re1] and [Re2] for the background information of hyperinterpolation on the sphere.

In spite of the best-order result (1.5), pointwise convergence (for arbitrary $f \in$ $C\left(\mathbb{S}^{d-1}\right)$ ) cannot be attained by hyperinterpolation. For this reason, Reimer [Re2] introduced the concept of generalized hyperinterpolation, whose definition is as follows. Suppose that

$$
D_{N}(t)=\sum_{k=0}^{N} a_{N, k} G_{k}(t), \quad N=0,1, \ldots,
$$

is a sequence of nonnegative polynomials on $[-1,1]$ satisfying

$$
\lim _{N \rightarrow \infty} a_{N, 0}=\lim _{N \rightarrow \infty} a_{N, 1}=1 .
$$


Then, the generalized hyperinterpolation operators associated to $\left\{D_{N}(t)\right\}_{N=0}^{\infty}$ are defined as

$G L_{N}(f)(x) \equiv G L_{N, D_{N}}(f)(x):=\sum_{\omega \in \Lambda_{N}} \lambda_{N, \omega} D_{N}(x \cdot \omega) f(\omega), f \in C\left(\mathbb{S}^{d-1}\right), x \in \mathbb{S}^{d-1}$.

Note that in the definition of $G L_{N}$ one assumes that the positive cubature formula involved is of degree $N+1$ rather than $2 N$. The point of generalized hyperinterpolation is that it can achieve a uniform convergence result for arbitrary $f \in C\left(\mathbb{S}^{d-1}\right)$ without increasing the cost of evaluation, as was shown by Reimer [Re2, Theorem 2].

An important example arises in generalized hyperinterpolation when one considers the Newman-Shapiro operators on $\mathbb{S}^{d-1}$, whose definition is given as follows. Let

$$
B_{2 N+1}(t)=B_{2 N}(t)=\gamma_{N+1}\left(\frac{G_{N+1}(t)}{t-\xi_{N+1}}\right)^{2}, \quad t \in[-1,1],
$$

where $\xi_{N+1}$ is the largest root of $G_{N+1}$, and the constant $\gamma_{N+1}$ is chosen so that

$$
\int_{\mathbb{S}^{d-1}} B_{2 N}(x \cdot y) d \sigma(y)=1, \quad x \in \mathbb{S}^{d-1} .
$$

The Newman-Shapiro operators are then defined by

$$
T_{N} f(x)=\int_{\mathbb{S}^{d-1}} f(y) B_{N}(x \cdot y) d \sigma(y), \quad x \in \mathbb{S}^{d-1}, \quad N=1,2, \ldots
$$

We denote by $G L_{N, B_{N}}$ the generalized hyperinterpolation operators associated to the kernels $B_{N}$. For the Newman-Shapiro operators $T_{N}$, Reimer [Re2, Theorem 7] proved that

$$
\left\|f-T_{N} f\right\|_{\infty} \leq C \omega_{2}\left(f, \frac{1}{N}\right), \quad f \in C\left(\mathbb{S}^{d-1}\right),
$$

where

$$
\begin{aligned}
& \omega_{2}(f, t)=\max \left\{\left|f_{u, v}(\alpha+t)-2 f_{u, v}(\alpha)+f_{u, v}(\alpha-t)\right|:\right. \\
& \left.\alpha \in \mathbb{R}, u, v \in \mathbb{S}^{d-1} \text { with } u \perp v\right\},
\end{aligned}
$$

is the modulus of smoothness of second order, and

$$
f_{u, v}(\varphi)=f(u \cos \varphi+v \sin \varphi), \text { for } \varphi \in \mathbb{R} \text { and } u, v \in \mathbb{S}^{d-1} \text { with } u \perp v ;
$$

while for the discrete operators $G L_{N, B_{N}}$, he proved [Re2, Theorem 6] that

$$
\left\|f-G L_{N, B_{N}} f\right\|_{\infty} \leq C \omega_{1}\left(f, N^{-1}\right), \quad f \in C\left(\mathbb{S}^{d-1}\right)
$$

where

$$
\omega_{1}(f, t)=\sup \left\{|f(x)-f(y)|: \quad x, y \in \mathbb{S}^{d-1} \text { and } d(x, y) \leq t\right\}
$$

is the first-order modulus of smoothness. It was asked by Reimer Re2] whether second-order results can be attained by generalized hyperinterpolation operators. Our main result (Theorem 1.1 below) in this paper gives an affirmative answer to this question. 
Theorem 1.1. Suppose that

$$
D_{N}(t)=\sum_{k=0}^{N} a_{N, k} G_{k}(t), \quad N=1,2, \ldots,
$$

is a sequence of polynomials on $[-1,1]$ satisfying

$$
\int_{\mathbb{S}^{d-1}} D_{N}(x \cdot y) d \sigma(y)=1, \quad x \in \mathbb{S}^{d-1}, \quad N=1,2, \ldots,
$$

and

$$
\sup _{N} \int_{0}^{\pi}(1+N \theta)^{2}\left|D_{N}(\cos \theta)\right| \sin ^{d-2} \theta d \theta \leq K<\infty .
$$

Let $G L_{N} \equiv G L_{N, D_{N}}$ denote the generalized hyperinterpolation operators defined by (1.6). Then for all $f \in C\left(\mathbb{S}^{d-1}\right)$, we have

$$
\left\|f-G L_{N} f\right\|_{\infty} \leq C K \omega_{2}\left(f, \frac{1}{N}\right),
$$

where $C>0$ depends only on $d$, and $\omega_{2}(f, t)$ is defined by (1.8).

Remark 1.1. It was shown in [Re2, pp. 197-199] that the kernels $B_{N}$ defined by (1.7) satisfy both of the conditions (1.9) and (1.10). Thus, by Theorem 1.1, (1.11) is true for the discrete operators $G L_{N, B_{N}}$.

Remark 1.2. The point of Theorem 1.1 is that we do not assume the positivity of the kernels $D_{N}$. The existence of a sequence of polynomials $D_{N}$ which satisfy both (1.9) and (1.10) but assume negative values on $[-1,1]$ is well known. See, for example, the sequence of polynomials $K_{[N / 2]}$ defined by (2.4) in Section 2 .

Remark 1.3. If, however, we assume that all the kernels $D_{N}$ are nonnegative, then we have the following.

Corollary 1.2. Suppose that

$$
D_{N}(t)=\sum_{k=0}^{N} a_{N, k} G_{k}(t), \quad N=1,2, \ldots,
$$

is a sequence of nonnegative polynomials on $[-1,1]$, and $G L_{N} \equiv G L_{N, D_{N}}(N=$ $1,2, \ldots)$ denote the generalized hyperinterpolation operators defined by (1.6). Then in order that

$$
\left\|f-G L_{N} f\right\|_{\infty} \leq C \omega_{2}\left(f, N^{-1}\right)
$$

(with $C>0$ depending only on $d$ ) holds for all $f \in C\left(\mathbb{S}^{d-1}\right)$, it is necessary and sufficient that

$$
a_{N, 0}=1
$$

and

$$
a_{N, 1} \geq 1-C_{d} N^{-2} .
$$

Corollary 1.2 can easily be deduced from Theorem 1.1 and the following two identities:

$$
\int_{\mathbb{S}^{d-1}} D_{N}(x \cdot y) d \sigma(y)=a_{N, 0}, \quad x \in \mathbb{S}^{d-1},
$$

and

$$
\int_{\mathbb{S}^{d-1}}(z \cdot y) D_{N}(x \cdot y) d \sigma(y)=a_{N, 1} z \cdot x, \quad z, x \in \mathbb{S}^{d-1} .
$$


The paper is organized as follows. In Section 2, we give an equivalent characterization of the regularity condition (1.4), which is in terms of the MarcinkiewitczZygmund inequality. Based on this characterization, we prove the main result in Section 3 .

\section{The REGULARITY CONDITION}

Our main purpose in this section is to give an equivalent characterization of the regularity condition of Sloan and Womersley [SW]. Our main result can be stated as follows.

Theorem 2.1. Suppose that $\Omega$ is a finite subset of $\mathbb{S}^{d-1},\left\{\mu_{\omega}: \omega \in \Omega\right\}$ is a set of positive numbers, and $n$ is a positive integer. If for some $0<p_{0}<\infty$ the inequality

$$
\sum_{\omega \in \Omega} \mu_{\omega}|f(\omega)|^{p_{0}} \leq C_{1} \int_{\mathbb{S}^{d-1}}|f(x)|^{p_{0}} d \sigma(x)
$$

with $C_{1}$ independent of $f$ holds for all $f \in \Pi_{n}^{d}$, then the following regularity condition must be satisfied:

$$
\sup _{x \in \mathbb{S}^{d-1}} \sum_{\omega \in \Omega \cap B\left(x, \frac{1}{n}\right)} \mu_{\omega} \leq C_{2} n^{-(d-1)},
$$

where $C_{2}=C C_{1}$ and $C>0$ depends only on $d$ and $p_{0}$.

Conversely, if the regularity condition (2.2) is satisfied for some constant $C_{2}>0$, then for any $0<p<\infty$ and any $f \in \Pi_{m}^{d}$ with $m \geq n$,

$$
\sum_{\omega \in \Omega} \mu_{\omega}|f(\omega)|^{p} \leq C C_{2}\left(\frac{m}{n}\right)^{d-1} \int_{\mathbb{S}^{d-1}}|f(y)|^{p} d \sigma(y),
$$

where $C>0$ depends only on $d$ and $p$.

We point out that while the second assertion of Theorem 2.1 with $p=1$ will be enough for the proof of our main result (Theorem 1.1) in this paper, we shall give the proof of the whole theorem in this section for the sake of completeness.

After this paper was completed, we learned that a result similar to Theorem 2.1 for $1 \leq p<\infty$ was obtained independently by $\mathrm{H}$. N. Mhaskar in a recent paper M2, Theorem 3.3].

For the proof of Theorem 2.1, we need three previously known lemmas. To state our first lemma, we let $\eta \in C^{\infty}[0, \infty)$ be such that $\eta(t)=1$ for $0 \leq t \leq 1$ and $\eta(t)=0$ for $t \geq 2$, and we define

$$
K_{N}(t) \equiv K_{N, \eta}(t)=\sum_{k=0}^{2 N} \eta\left(\frac{k}{N}\right) G_{k}(t), \quad t \in[-1,1], \quad N=1,2, \ldots
$$

By (1.1) and (1.2), it follows that for very $f \in \Pi_{N}^{d}$

$$
f(x)=\int_{\mathbb{S}^{d-1}} f(y) K_{N}(x \cdot y) d \sigma(y), \quad x \in \mathbb{S}^{d-1} .
$$

We will keep the function $\eta$ and the notation $K_{N}$ for the rest of this section. Our first lemma, which was proved in $\mathrm{BD}$, Lemma 3.3], can be stated as follows. 
Lemma 2.2. For $\theta \in[0, \pi]$ and any positive integer $\ell$, we have

$$
\left|K_{N}^{(i)}(\cos \theta)\right| \leq C_{\ell, i} N^{d-1+2 i} \min \left\{1,(N \theta)^{-\ell}\right\}, \quad i=0,1, \ldots, N=1,2, \ldots,
$$

where $K_{N}^{(0)}(t)=K_{N}(t), K_{N}^{(i)}(t)=\left(\frac{d}{d t}\right)^{i}\left\{K_{N}(t)\right\}$ for $i \geq 1$, and the constant $C_{\ell, i}$ depends only on $\ell, i$ and $\eta$.

The point of Lemma 2.2 is that the positive integer $\ell$ can be chosen arbitrarily big. An estimate analogous to Lemma 2.2 was obtained independently by H. N. Mhaskar in a recent paper [M1, Lemma 4.10].

Our second lemma, Lemma 2.3 below, was proved by Q. T. Le Gia and I. H. Sloan in [LS, Lemma 5.1].

Lemma 2.3. Suppose that $\Omega$ is a finite subset of $\mathbb{S}^{d-1},\left\{\mu_{\omega}: \omega \in \Omega\right\}$ is a set of positive numbers satisfying the regularity condition (2.2) for some $C_{2}>0$. Then we have

(a) For every spherical cap $B(x, \alpha)$ with radius $\alpha \geq \frac{1}{n}$,

$$
\sum_{\omega \in \Omega \cap B(x, \alpha)} \mu_{\omega} \leq C C_{2}|B(x, \alpha)|,
$$

where $C>0$ depends only on $d$.

(b) For every spherical collar $B(x, \alpha, \alpha+\beta)$ with spherical height $\beta \geq \frac{1}{n}$,

$$
\sum_{\omega \in B(x, \alpha, \alpha+\beta) \cap \Omega} \mu_{\omega} \leq C C_{2}|B(x, \alpha, \alpha+\beta)|,
$$

where $C>0$ depends only on $d$.

Our final lemma, Lemma 2.4 below, is well known (see, for instance, BDS, SW] or [MNW]).

Lemma 2.4. For each positive integer $N$, there exists a positive cubature formula of degree $4 N$ on $\mathbb{S}^{d-1}$, that is,

$$
\int_{\mathbb{S}^{d-1}} f(y) d \sigma(y)=\sum_{j=1}^{M_{N}} w_{j, N} f\left(t_{j, N}\right), \quad \text { for all } f \in \Pi_{4 N}^{d},
$$

where $t_{j, N} \in \mathbb{S}^{d-1}, 0<w_{j, N} \leq C_{d} N^{-(d-1)}$, and $M_{N} \sim N^{d-1}$.

Proof of Theorem 2.1. First, we assume (2.1) is true for some $0<p_{0}<\infty$, all $f \in \Pi_{n}^{d}$ and for some finite set $\Omega \subset \mathbb{S}^{d-1}$, and we will prove the regularity condition (2.2). Let

$$
P_{[n / 2]}(\cos \theta)=\frac{P_{[n / 2]}^{\left(\frac{d}{p_{0}}-\frac{1}{2},-\frac{1}{2}\right)}(\cos \theta)}{P_{[n / 2]}^{\left(\frac{d}{p_{0}}-\frac{1}{2},-\frac{1}{2}\right)}(1)}, \quad \theta \in[0, \pi],
$$

where $P_{k}^{(\alpha, \beta)}$ denotes the Jacobi polynomial of degree $k$ and with indices $\alpha, \beta$, as

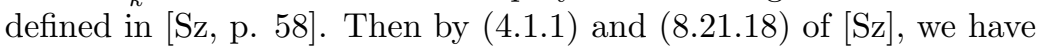

$$
\left|P_{[n / 2]}(\cos \theta)\right| \leq \min \left\{1, C_{d, p_{0}}(n \theta)^{-d / p_{0}}\right\}, \quad \theta \in[0, \pi] .
$$

Thus, using Bernstein's inequality for trigonometric polynomials, we have, for all $\theta \in[0, \pi]$,

$$
\left|P_{[n / 2]}(\cos \theta)-1\right|=\left|P_{[n / 2]}(\cos \theta)-P_{[n / 2]}(1)\right| \leq \frac{n \theta}{2}\left\|P_{[n / 2]}\right\|_{\infty}=\frac{n \theta}{2},
$$


which implies that for $0 \leq \theta \leq \frac{1}{n}$,

$$
\frac{1}{2} \leq P_{[n / 2]}(\cos \theta) \leq 1
$$

Now, for any fixed $x \in \mathbb{S}^{d-1}$, using (2.1) with $f(y)=P_{[n / 2]}(y \cdot x)$, we deduce

$$
\sum_{\omega \in \Omega \cap B\left(x, \frac{1}{n}\right)} \mu_{\omega} \leq 2^{p_{0}} \sum_{\omega \in \Omega} \mu_{\omega}\left|P_{[n / 2]}(x \cdot \omega)\right|^{p_{0}} \leq 2^{p_{0}} C_{1} \int_{\mathbb{S}^{d-1}}\left|P_{[n / 2]}(x \cdot y)\right|^{p_{0}} d \sigma(y),
$$

which, by (2.7) and a straightforward calculation, is controlled by $C_{1} C_{d, p_{0}} n^{-(d-1)}$. This proves (2.2) with $C_{2}=C_{d, p_{0}} C_{1}$.

Next, we prove the second assertion of Theorem 2.1. Suppose that the regularity condition (2.2) is satisfied. We will prove that (2.3) holds for all $f \in \Pi_{m}^{d}$ with $m \geq n$. Set

$$
A_{m}(\theta)=m^{d-1}(1+m \theta)^{-d}, \quad \theta \in[0, \pi] .
$$

The following estimate will play a crucial role in our proof:

$$
\max _{x \in \mathbb{S}^{d-1}} \sum_{\omega \in \Omega} \mu_{\omega} A_{m}(d(\omega, x)) \leq C C_{2}\left(\frac{m}{n}\right)^{d-1},
$$

where $C>0$ depends only on $d$ and $p$ when $p$ is small.

For the moment, we take (2.9) for granted and proceed with the proof. We start with the equation

$$
f(x)=\int_{\mathbb{S}^{d-1}} f(y) K_{m}(x \cdot y) d \sigma(y), \quad x \in \mathbb{S}^{d-1}, \quad f \in \Pi_{m}^{d},
$$

and consider the following two cases:

Case $1.1 \leq p<\infty$.

In this case, using (2.5) and Hölder's inequality, we have, for $f \in \Pi_{m}^{d}$ and all $x \in \mathbb{S}^{d-1}$,

$$
|f(x)|^{p} \leq\left[\int_{\mathbb{S}^{d-1}}|f(y)|^{p}\left|K_{m}(x \cdot y)\right| d \sigma(y)\right]\left[\int_{\mathbb{S}^{d-1}}\left|K_{m}(x \cdot y)\right| d \sigma(y)\right]^{p-1} .
$$

Therefore, by Lemma 2.2 applied to $\ell=d$ and $i=0$, and a straightforward calculation, it follows that for $f \in \Pi_{m}^{d}$ and all $x \in \mathbb{S}^{d-1}$

$$
|f(x)|^{p} \leq C \int_{\mathbb{S}^{d-1}}|f(y)|^{p} A_{m}(d(x, y)) d \sigma(y),
$$

where $A_{m}$ is defined by (2.8). Now combining this last inequality with (2.9), we obtain

$$
\sum_{\omega \in \Omega} \mu_{\omega}|f(\omega)|^{p} \leq C\|f\|_{p}^{p} \max _{y \in \mathbb{S}^{d-1}}\left[\sum_{\omega \in \Omega} \mu_{\omega} A_{m}(d(\omega, y))\right] \leq C C_{2}\left(\frac{m}{n}\right)^{d-1}\|f\|_{p}^{p} .
$$

Case 2. $0<p<1$.

In this case, we use (2.10) and the cubature formulas in Lemma 2.4 to obtain

$$
f(x)=\sum_{j=1}^{M_{m}} w_{j, m} f\left(\rho t_{j, m}\right) K_{m}\left(x \cdot \rho t_{j, m}\right), \quad x \in \mathbb{S}^{d-1}, \quad \rho \in S O(d),
$$


where $f \in \Pi_{m}^{d}, S O(d)$ denotes the group of rotations on $\mathbb{S}^{d-1}$. Since $0<w_{j, m} \leq$ $C_{d} m^{-(d-1)}$, it follows by Lemma 2.2 with $\ell=\frac{d}{p}$ that for $x \in \mathbb{S}^{d-1}$ and $\rho \in S O(d)$,

$$
|f(x)|^{p} \leq C \sum_{j=1}^{M_{m}} m^{-(d-1) p}\left|f\left(\rho t_{j, m}\right)\right|^{p} m^{(d-1)(p-1)} A_{m}\left(d\left(x, \rho t_{j, m}\right)\right) .
$$

This implies that

$$
\begin{aligned}
\sum_{\omega \in \Omega} \mu_{\omega}|f(\omega)|^{p} & \leq C m^{-(d-1)}\left[\inf _{\rho \in S O(d)} \sum_{j=1}^{M_{m}}\left|f\left(\rho t_{j, m}\right)\right|^{p}\right]\left[\max _{y \in \mathbb{S}^{d-1}} \sum_{\omega \in \Omega} \mu_{\omega} A_{m}(d(\omega, y))\right] \\
& \left.\leq C C_{2}\left(\frac{m}{n}\right)^{d-2} m^{-(d-1)} \sum_{j=1}^{M_{m}} \int_{S O(d)}\left|f\left(\rho t_{j, m}\right)\right|^{p} d \rho \quad \text { (by (2.9) }\right) \\
& \left.\leq C C_{2}\left(\frac{m}{n}\right)^{d-2}\|f\|_{p}^{p}, \quad \text { (by the fact that } M_{m} \sim m^{d-1}\right),
\end{aligned}
$$

where $d \rho$ denotes the usual Haar measure on the group $S O(d)$.

Therefore, in either case, we have proven the desired inequality (2.3), taking (2.9) for granted.

It remains to prove (2.9). The proof is based on Lemma 2.3. In fact, using Lemma 2.3 and the definition of $A_{m}$, we have, for any $x \in \mathbb{S}^{d-1}$,

$$
\begin{aligned}
& \sum_{\omega \in \Omega} \mu_{\omega} A_{m}(d(x, \omega)) \\
& \leq m^{d-1} \sum_{\omega \in \Omega \cap B\left(x, \frac{1}{n}\right)} \mu_{\omega}+\sum_{k=1}^{n-1} \sum_{\omega \in B(x, k / n,(k+1) / n) \cap \Omega} \mu_{\omega} m^{d-1}\left(\frac{m k}{n}\right)^{-d} \\
& \quad+m^{-1} \sum_{\omega \in B(-x, \pi-1) \cap \Omega} \mu_{\omega} \\
& \leq C C_{2} m^{d-1}\left|B\left(x, \frac{1}{n}\right)\right|+C C_{2} m^{-1} \sum_{k=1}^{n-1}\left(\frac{n}{k}\right)^{d}|B(x, k / n,(k+1) / n)| \\
& \leq C C_{2}\left(\frac{m}{n}\right)^{d-1}, \quad+C C_{2} m^{-1}|B(-x, \pi-1)|
\end{aligned}
$$

since $|B(x, \alpha)| \sim \alpha^{d-1}$ and $|B(x, k / n,(k+1) / n)| \sim k^{d-2} / n^{d-1}$ for $1 \leq k \leq n-1$. This proves (2.9) and hence completes the proof of Theorem 2.1 .

\section{Proof of Theorem 1.1}

For the proof of Theorem 1.1, we need three lemmas. The first two lemmas are previously known.

Lemma $3.1(\mathbb{N}])$. Suppose that $T$ is a continuously differentiable, $2 \pi$-periodic function on $\mathbb{R}$ with $\int_{0}^{2 \pi} T(x) d x=0$. Then

$$
\|T\|_{C(\mathbb{T})} \leq C\left\|T^{\prime}\right\|_{C(\mathbb{T})},
$$

where $C>0$ is an absolute constant, and throughout this section, $\|\cdot\|_{C(\mathbb{T})}$ is defined as $\|T\|_{C(\mathbb{T})}=\max _{x \in[0,2 \pi]}|T(x)|$. 
Lemma 3.2 (St] $)$. If $T_{n}$ is a trigonometric polynomial of degree at most $n$ and $r \geq 1$ is a positive integer, then for $0<h<\frac{2 \pi}{n}$,

$$
\left\|T_{n}^{(r)}\right\|_{C(\mathbb{T})} \leq\left(\frac{n}{2 \sin (n h / 2)}\right)^{r}\left\|\triangle_{h}^{r} T_{n}\right\|_{C(\mathbb{T})},
$$

where

$$
\triangle_{h}^{r} T_{n}(x)=\sum_{j=0}^{r}(-1)^{r-j}\left(\begin{array}{l}
r \\
j
\end{array}\right) T_{n}(x+j h) .
$$

Lemma 3.3. Suppose that $D_{N}$ satisfies the assumptions in Theorem 1.1, and $G L_{N} \equiv G L_{N, D_{N}}$ is defined by (1.6). Then for $f \in C\left(\mathbb{S}^{d-1}\right)$, we have

$$
\left\|G L_{N} f\right\|_{\infty} \leq C K\|f\|_{\infty}
$$

where $C>0$ depends only on $d$.

Proof. By the definition (1.6), we have, for $f \in C\left(\mathbb{S}^{d-1}\right)$ and $x \in \mathbb{S}^{d-1}$,

$$
\begin{aligned}
\left|G L_{N} f(x)\right| & \leq\left(\sum_{\omega \in \Lambda_{N}} \lambda_{N, \omega}\left|D_{N}(x \cdot \omega)\right|\right)\|f\|_{\infty} \\
& \leq C\left(\int_{\mathbb{S}^{d-1}}\left|D_{N}(x \cdot y)\right| d \sigma(y)\right)\|f\|_{\infty} \quad(\text { by (1.4) } \text { and Theorem 2.1) } \\
& \leq C K\|f\|_{\infty} \quad(\text { by (1.10) }) .
\end{aligned}
$$

This completes the proof.

Proof of Theorem 1.1. Let $g \in \Pi_{N}^{d}$ be such that

$$
\|f-g\|_{\infty}=E_{N}(f)_{\infty}:=\inf _{p \in \Pi_{N}^{d}}\|f-p\|_{\infty} .
$$

Then by Lemma 3.3, we have

$$
\begin{aligned}
\left\|f-G L_{N}(f)\right\|_{\infty} & \leq\left\|G L_{N}(f-g)\right\|_{\infty}+\left\|G L_{N}(g)-g\right\|_{\infty}+E_{N}(f)_{\infty} \\
& \leq C K E_{N}(f)_{\infty}+\left\|G L_{N}(g)-g\right\|_{\infty} .
\end{aligned}
$$

It will be shown that

$$
\left\|g-G L_{N}(g)\right\|_{\infty} \leq C K \omega_{2}\left(g, \frac{1}{N}\right)
$$

Once (3.2)) is proved, then by (3.1) and the Jackson-type estimate (see, for example, [Re2, Theorem 7]), we obtain

$$
\left\|f-G L_{N}(f)\right\|_{\infty} \leq C K E_{N}(f)_{\infty}+C K \omega_{2}\left(f-g, \frac{1}{N}\right)+C K \omega_{2}\left(f, \frac{1}{N}\right) \leq C K \omega_{2}\left(f, \frac{1}{N}\right),
$$

which gives the desired inequality (1.11). Thus, the proof of Theorem 1.1 is reduced to the proof of (3.2)). $\mathbb{S}^{d-1}$,

To show (3.2), we claim that for $x=\left(x_{1}, \ldots, x_{d}\right) \in \mathbb{S}^{d-1}$ and $y=\left(y_{1}, \ldots, y_{d}\right) \in$

$$
\left|g(y)-g(x)-\sum_{j=1}^{d} g_{j}^{\prime}(x)\left(y_{j}-x_{j}(x \cdot y)\right)\right| \leq C N^{2}(1-x \cdot y) \omega_{2}\left(g, \frac{1}{N}\right),
$$

where $g_{j}^{\prime}(x)=\frac{\partial G(x)}{\partial x_{j}}$ and $G(z)=g\left(\frac{z}{|z|}\right)$ for $z \in \mathbb{R}^{d} \backslash\{0\}$. 
For the proof of the claim (3.3), we assume that $y=x \cos \theta+u \sin \theta$, where $\theta=d(x, y)$ and $u=\left(u_{1}, \ldots, u_{d}\right) \in \mathbb{S}^{d-1}$ with $u \perp x$. We set

$$
g_{x, u}(\varphi)=g(x \cos \varphi+u \sin \varphi), \quad \varphi \in \mathbb{R} .
$$

Then $g_{x, u}$ is a trigonometric polynomial of degree at most $N$, and moreover,

$$
g_{x, u}^{\prime}(\varphi)=\sum_{j=1}^{d} g_{j}^{\prime}(x \cos \varphi+u \sin \varphi)\left(-x_{j} \sin \varphi+u_{j} \cos \varphi\right) .
$$

It follows that

$$
\begin{aligned}
I: & \equiv\left|g(y)-g(x)-\sum_{j=1}^{d} g_{j}^{\prime}(x)\left(y_{j}-x_{j}(x \cdot y)\right)\right| \\
& =\left|g_{x, u}(\theta)-g_{x, u}(0)-\left(\sum_{j=1}^{d} g_{j}^{\prime}(x) u_{j}\right) \sin \theta\right| \\
& \leq\left|g_{x, u}(\theta)-g_{x, u}(0)-g_{x, u}^{\prime}(0) \theta\right|+C\left\|g_{x, u}^{\prime}\right\|_{C(\mathbb{T})} \theta^{3} \\
& =\frac{\theta^{2}}{2}\left|g_{x, u}^{\prime \prime}(\xi)\right|+C \theta^{3}\left\|g_{x, u}^{\prime}\right\|_{C(\mathbb{T})}, \quad \text { for some } \xi \in[0, \theta] .
\end{aligned}
$$

However, noting that

$$
\int_{0}^{2 \pi} g_{x, u}^{\prime}(t) d t=g_{x, u}(2 \pi)-g_{x, u}(0)=0
$$

using Lemma 3.1, we deduce

$$
\theta^{3}\left\|g_{x, u}^{\prime}\right\|_{C(\mathbb{T})} \leq C \theta^{2}\left\|g_{x, u}^{\prime \prime}\right\|_{C(\mathbb{T})}, \quad \theta \in[0, \pi]
$$

Therefore, combining this last inequality with (3.4), we obtain

$$
\begin{aligned}
I & \leq C \theta^{2}\left\|g_{x, u}^{\prime \prime}\right\|_{C(\mathbb{T})} \\
& \leq C N^{2}(1-x \cdot y)\left\|\triangle_{N^{-1}}^{2}\left(g_{x, u}\right)\right\|_{C(\mathbb{T})} \quad \text { (by Lemma 3.2) } \\
& \leq C N^{2}(1-x \cdot y) \omega_{2}\left(g, \frac{1}{N}\right) \quad(\text { by the definition (1.8) }),
\end{aligned}
$$

which proves the claim (3.3)).

Now we are ready to prove (3.2). By (1.9) and the definition (1.6), we have

$$
G L_{N}(g)(x)-g(x)=\sum_{\omega \in \Lambda_{N}} \lambda_{N, \omega}(g(\omega)-g(x)) D_{N}(x \cdot \omega) .
$$

We note that for each $1 \leq j \leq d$,

$$
\begin{gathered}
\sum_{\omega=\left(\omega_{1}, \ldots, \omega_{d}\right) \in \Lambda_{N}}\left(\omega_{j}-x_{j}(x \cdot \omega)\right) \lambda_{N, \omega} D_{N}(x \cdot \omega) \\
=\int_{\mathbb{S}^{d-1}} D_{N}(x \cdot y)\left(y_{j}-x_{j}(x \cdot y)\right) d \sigma(y) \\
=a_{N, 1}\left(x_{j}-x_{j}(x \cdot x)\right)=0,
\end{gathered}
$$

where the first equality follows by the assumption that the cubature formula $Q_{N}$ is of degree $N+1$, while the second follows from the fact that $h_{j}(y)=y_{j}-x_{j}(x \cdot y)$ 
is a spherical harmonic of degree 1 . Therefore, using the claim (3.3), we conclude that for $x \in \mathbb{S}^{d-1}$,

$$
\begin{aligned}
& \left|G L_{N}(g)(x)-g(x)\right| \leq C N^{2} \omega_{2}\left(g, N^{-1}\right) \sum_{\omega \in \Lambda_{N}} \lambda_{N, \omega}(1-x \cdot \omega)\left|D_{N}(x \cdot \omega)\right| \\
& \quad \leq C N^{2} \omega_{2}\left(g, N^{-1}\right) \int_{\mathbb{S}^{d-1}}(1-x \cdot y)\left|D_{N}(x \cdot y)\right| d \sigma(y),
\end{aligned}
$$

(by (1.4) and Theorem 2.1)

$$
\leq C K \omega_{2}\left(g, N^{-1}\right) \quad(\text { by }(1.10))
$$

which proves (3.2) and hence completes the proof of Theorem 1.1.

\section{ACKNOWLEDGEMENT}

The author expresses his deep gratitude to an anonymous referee for reading this paper carefully and giving many helpful comments on it.

\section{REFERENCES}

[BD] G. Brown and F. Dai, Approximation of smooth functions on compact two-point homogeneous spaces, J. Funct. Anal. 220 (2005), no. 2, 401-423. MR2119285

[BDS] G. Brown, F. Dai and Y. Sh. Sun, Kolmogorov width of classes of smooth functions on the sphere $\mathbb{S}^{d-1}$, J. Complexity 18 (2002), no. 4, 1001-1023. MR1933699 (2003g:41039)

[LS] Q. T. Le Gia and I. H. Sloan, The uniform norm of hyperinterpolation on the unit sphere in an arbitrary number of dimensions, Constr. Approx. 17 (2001), no. 2, 249-265. MR.1814361 (2001m:41021)

[M1] H. N. Mhaskar, Polynomial operators and local smoothness classes on the unit interval, J. Approx. Theory 131 (2004), no. 2, 243-267. MR2106540

[M2] H. N. Mhaskar, Weighted quadrature formulas and approximation by zonal function networks on the sphere, Preprint.

[MNW] H. N. Mhaskar and F.J. Narcowich and J.D. Ward, Spherical Marcinkiewicz-Zygmund inequalities and positive quadrature, Math. Comp. 70 (2001), 1113-1130. MR1710640 (2002a:41032)

[N] D. G. Northcott, Some inequalities between periodic functions and their derivatives, J. London Math. Soc. 14 (1939), 198-202. MR0000417 (1:71c)

[Re1] M. Reimer, Hyperinterpolation on the sphere at the minimal projection order, J. Approx. Theory 104 (2000), no. 2, 272-286. MR1761902 (2001c:41031)

[Re2] M. Reimer, Generalized hyperinterpolation on the sphere and the Newman-Shapiro operators, Constr. Approx. 18 (2002), no. 2, 183-203. MR.1890495 (2003a:41005)

[Sl] I. H. Sloan, Polynomial interpolation and hyperinterpolation over general regions, J. Approx. Theory 83 (1995), no. 2, 238-254. MR1357589 (96h:41036)

[SW] I. H. Sloan and R. S. Womersley, Constructive polynomial approximation on the sphere, J. Approx. Theory 103 (2000), no. 1, 91-118. MR.1744380 (2000k:41009)

[St] S. B. Stečkin, A generalization of some inequalities of S. N. Bernštern (Russian), Doklady Akad. Nauk SSSR (N.S.) 60 (1948), 1511-1514. MR0024993 (9:579g)

[Sz] G. Szegö, Orthogonal polynomials, American Mathematical Society Colloquium Publications 23, Fourth edition, American Mathematical Society, Providence, RI, 1975. MR $0372517(51: 8724)$

Department of Mathematical and Statistical Sciences, University of Alberta, Edmonton, Alberta, Canada T6G 2G1

E-mail address: dfeng@math.ualberta.ca 